\title{
Correlation between behaviorally measured comfort $C$ levels and electrically evoked stapedius reflex thresholds (ESRT) in children with unilateral cochlear implant
}

\begin{abstract}
Introduction: Decrease in the lower age limit for paediatric cochlear implantation and increasing records of cochlear implantation in children with associated impairments demand quick and easily available objective measurements to provide MAP in these children as their speech and language ability may not be adequate to provide clear cut explanation about comfort level. Providing adequate amount of acoustic stimulation in this golden period of life (i.e. 0-2 years) is important in ensuring the proper utilization of acoustic cues. Findings of adult population cannot be directly applied to younger children and till date there is no Indian study reporting relationship between Electrically Evoked Stapedial Reflex Thresholds (ESRT) and behavioural Comfort levels, especially in pediatric population.
\end{abstract}

Aim: The present study aimed to assess the correlation between behaviorally measured comfort (C) levels and Electrically Evoked Stapedius Reflex Thresholds (ESRT) in children with unilateral cochlear implant.

Method: Children $(n=40)$ with age range of 4-12 years, who received the same type of $\mathrm{CI}$ and electrode configuration, were selected for the study. Participants were divided into two groups - younger and older - based on their chronological age. Behavioural C-level measurement and ESRT measurement were done after tympanometry for both the groups. Correlation between the two measures was obtained and comparison between the two groups was done.

Results and discussion: Recorded ESRT was consistently higher than the behaviorally obtained C-level for both the groups. The correlation between ESRT and C-level varies from moderate to very strong and strong to very strong for the younger and older groups respectively. No statistically significant difference was found between the correlations for the two age groups.

Conclusions: High correlations were found between the ESRT and behaviorally obtained C-levels. Thus ESRT can be utilized as a tool for programming in younger implant recipients

Keywords: C-level, ESRT, unilateral cochlear implant, correlation
Volume II Issue 4 - 2019

\section{Roshani Mishra,' Aparna Nandurkar ${ }^{2}$ \\ 'Practicing Audiologist and Speech-Language Pathologist, India ${ }^{2}$ Department of Audiology, Ali Yavar Jung National Institute of Speech and Hearing Disabilities, India}

Correspondence: Aparna Nandurkar, Lecturer (Speech and Hearing), Department of Audiology, Ayjnishd, Bandra Reclamation, India, Tel +919820446304,

Email aparnanitinnandurkar@gmail.com

Received: July 31, 2019 | Published: August 09, 2019
Abbreviations: ESRT, evoked stapedial reflex thresholds; CI, cochlear implantation; BOA, behavioural observation audiometry; VRA, visual reinforcement audiometry; ECAP, electrically evoked compound action potential; NRT, neural response telemetry; EABR, electrically evoked auditory brain stem response

\section{Introduction}

Currently Cochlear Implantation (CI) has become the best possible hearing solution for children and adults with severe to profound sensori-neural hearing loss who cannot be helped optimally with conventional hearing aids. ${ }^{1}$ Cochlear Implant is a surgically implanted electronic device which consists of two components 1) External including a microphone, connecting cables, speech processor and transmitting coil and 2) Internal - consisting of receiver-stimulator package and electrode array. The microphone picks up the sounds from the environment and sends it to the speech processor. The speech processor codes the signal for transmission to the internal device and sends it to the transmitting coil via connecting cable. Internal receiver coil receives the information and decodes the signal and delivers electrical stimulation to the implanted electrodes. ${ }^{2}$

After surgery, when the recipient's wound is healed completely, that is approximately 2 to 3 weeks post implantation, a program or map is provided to the client. It is done by providing an appropriate minimum and maximum amount of current for the stimulation of electrodes. It is generally created by setting Threshold level or Tlevel, which is the minimum amount of electrical stimulation required for the auditory system to perceive sound and Comfort level or Clevel, which is the higher limit of electrical stimulation judged to be most comfortable or loud but comfortable. ${ }^{3}$

There are two methods to program cochlear implants in children:

Subjective methods: These include use of behavioural responses obtained from the child to set the T and C-levels. Based on the age and the ability of the child to co-operate various behavioural responses 
such as those used during hearing testing can be employed. These may include responses like those used in Behavioural Observation Audiometry (BOA), Visual Reinforcement Audiometry (VRA), and Conditioned Play Audiometry (CPA).

Objective methods: These include use of electrophysiological measures to set the $\mathrm{T}$ and C-levels. The commonly used objective measures for programming the cochlear implant are:

a. Electrically evoked compound action potential (ECAP): An action potential is the response which is obtained from neural elements. In reference to cochlear implantation, action potentials from the peripheral auditory nerve can be measured in response to stimulation of intra-cochlear electrode which activates the auditory nerve and ECAP is generated. Voltage at second intracochlear electrode (i.e. recording electrode) is sampled, amplified and transmitted back to the externally worn coil. For Nucleus implants, separate software is available to measure the ECAP which is known as Neural Response Telemetry (NRT). Brown et al., ${ }^{4}$ found that there is correlation of 0.54 and 0.56 between $\mathrm{T}$ level and C-level respectively and the ECAP threshold.

b. Electrically evoked auditory brain stem response (EABR): It utilizes the response of the neural element to electrical stimulation through the cochlear implant. It is a rarely used procedure, because it is time consuming and requires external recording equipment, scalp electrodes and sedation. Results of EABR are more prone to artifacts. ${ }^{3}$

c. Electrically evoked stapedius reflex threshold (ESRT): A stapedius reflex is the contraction of the stapedius muscle in response to loud sounds. In normal hearing persons, the reflex is elicited bilaterally in response to acoustic stimulation in either ear. It can be measured by using standard tympanometer. ${ }^{5}$ There are two types of stapedius reflexes: 1) Ipsilateral (Uncrossed) ReflexWhen signal is presented in the left ear and a reflex is measured in that ear, it is referred as left uncrossed reflex. 2) Contralateral (Crossed) reflex- When a signal is presented to the left ear and a reflex is measured in the right ear; it is referred to as a left crossed reflex. ${ }^{6}$ In cochlear implant recipients, stapedius reflex could be measured in the contra- lateral (Non-implanted) ear via electrical stimulation in the implant. An Electrically Evoked Stapedius Reflex Threshold (ESRT) is defined as the lowest level of electrical stimulation that elicits a measurable response. ${ }^{5}$

Sometimes it becomes a difficult task even for an experienced audiologist to get accurate feedback from infants and children while programming the cochlear implant. Inaccurate programming of the device can in turn lead to overstimulation. Overstimulation can lead to non-acceptance of the device in case of children and has also been reported to be associated with echoing and wavering sound quality by patients. Mertes et al., ${ }^{3}$ reported number of cases where overstimulation led to unclear speech and lag in expressive language development in cochlear implant users. However similar description has given by patients when C-levels are set too low. Therefore, it is important to set the C-levels at an adequate level.

Recipient's focused participation is required while mapping to set $\mathrm{T}$ and C-levels accurately. T-levels can be adequately set by training the child to respond to soft stimuli and obtaining minimum response levels. It is, however, difficult for children with pre-lingual hearing loss to understand the concept of comfort level and to accurately provide feedback about comfortable levels, primarily due to their inadequate language abilities and complex nature of loudness description. Therefore, clinicians may utilize objective procedures which do not require active participation of the cochlear implant recipient to find out $\mathrm{T}$ and $\mathrm{C}$-levels. ESRT is a comparatively less time-consuming procedure and requires only additional clinical immittance meter and cochlear implant mapping software and hence may prove to be useful in mapping young children. There have been very limited studies focused on use of ESRT in mapping young children with cochlear implant in the Indian context. Such a study will help in programming young children more effectively with the use of this easily available objective measure.

\section{Need for the study}

Most of the studies on correlation between behavioural comfort levels and objective ESRT levels have been done in foreign countries and they mainly focus on intra-operative monitoring of ESR. Limited numbers of studies have discussed about the postoperative ESRT measurement and its correlation with behavioural C-level measurement and are mainly based on adult population. Decrease in the lower age limit for paediatric cochlear implantation and increasing records of cochlear implantation in children with associated impairments demand quick and easily available objective measurement to provide MAP in these children as their current speech and language ability is not adequate to provide clear cut explanation about comfort level. Providing adequate amount of acoustic stimulation in this golden period of life (i.e. 0-2 years) is important in ensuring the proper utilization of acoustic cues. As findings of adult population cannot be directly applied to younger children and till date there is no Indian study reporting correlation of post-operative ESRT with behavioural C-level measurement, this study is proposed.

\section{Aim}

The present study aimed to assess the correlation between behaviorally measured comfort (C) levels and Electrically Evoked Stapedius Reflex Thresholds (ESRT) in children with unilateral Cochlear Implant. Further, the study aimed to find out if the correlation between the behavioural $\mathrm{C}$ levels and ESRT measures was significantly differed for younger and older children. As older children's understanding and ability to respond to Comfort levels is expected to be better than that for younger children it would be interesting to compare the two groups.

\section{Material and methods}

Sample: The sample consisted of 40 participants who were further divided into two groups based on their chronological age - Group A included children between 4 to 7 years and Group B included children between 8 and 12 years. 20 participants were included in each group. All participants were implanted with Freedom CI24 RE (ST) device and used Freedom $(\mathrm{N}=22)$ or $\mathrm{CP} 802(\mathrm{~N}=18)$ as their external Speech Processor. Only participants in whom ESRT was present at all the three electrodes specified for the study (Number 1, 11, \& 22) were included. There were other five children in whom measureable ESRT was not obtained for all specified electrodes. So, they were excluded from the study. Among these excluded children, no ESRT was obtained for 4 children till the maximum level of current stimulation for all three electrodes whereas 1 child reported discomfort during measurement of ESRT at electrode number 1. Table 1 shows the details of the participants included in the two groups. The specific inclusion criteria were as stated below. 
Table I Details of the participants

\begin{tabular}{|c|c|c|c|c|c|c|c|}
\hline \multirow[t]{2}{*}{ Group } & \multirow[t]{2}{*}{$\mathbf{N}$} & \multicolumn{2}{|c|}{$\begin{array}{l}\text { Chronological } \\
\text { age (months) }\end{array}$} & \multicolumn{2}{|c|}{$\begin{array}{l}\text { Implant age } \\
\text { (months) }\end{array}$} & \multicolumn{2}{|c|}{ Processor } \\
\hline & & Mean & SD & Mean & SD & Freedom & CP802 \\
\hline$A(4-7 Y)$ & $20(8 \mathrm{~F}, \mathrm{I} 2 \mathrm{M})$ & 67.45 & \pm 9.44 & 24.05 & \pm 10.77 & 15 & 05 \\
\hline$B(8-I 2 Y)$ & 20 (IOF,IOM) & 110.7 & \pm|| .93 & 52.5 & \pm 20.74 & 07 & 13 \\
\hline
\end{tabular}

\section{Inclusion criteria}

i. Children aged 4 to 7 years for group A

ii. Children aged 8 to 12 years for group B

iii. Pre-lingual bilateral severe to profound SN hearing loss

iv. Unilateral Cochlear Implantation

v. No presence of Middle Ear Pathology as ascertained with Bilateral Type "A" Tympanograms

vi. Minimum of 1 year post switch-on

vii. Anatomically normal cochlea and auditory nerve

viii. Complete insertion of cochlear electrodes

ix. Mapped using default parameters

x. No associated impairments such as visual impairment, ADHD, Autism spectrum disorder, mental retardation, developmental delay, cerebral palsy

xi. Ability to provide consistent behavioural response for obtaining comfort levels

\section{Equipment and other accessories}

a. Immitance Audiometer (GSI Grason- stadler Tympstar pro) ${ }^{7}$

b. Custom Sound $5.1^{8}$ programming software for Nucleus implants with Programming Pod (N530) and Freedom and CP802 programming cables

c. Custom Sound EP $5.1^{8}$ software

d. Laptop (Acer)- Model Number- E5-511.

\section{Procedure}

Consent: Informed written consent in the language comfortable to them was taken from the parents and verbal assent was obtained from all children. Case History: Primary demographic details, details of CT scan \& MRI and details of the cochlear implantation were obtained from the parents in a prescribed format.

\section{C-level measurement}

Behavioural C- level was measured during programming session by asking the recipient to indicate the perceptual loudness level of the stimulus in terms of a) Too Soft, b) Alright, c) Too Loud. The concept of soft and loud was clearly explained to the children with the help of pictures, facial expressions, written words, etc. as necessary. All verbal and non-verbal (gestural, picture pointing, facial expression, body language) responses were taken in consideration. The level at which the child responded as "Alright" loudness has been considered as the behavioural C-level. C-levels for electrode numbers 1, 11 and
22 were recorded on participant data collection sheet

Tympanometry: Tympanometry was done to ascertain normal middle ear status by using the $226 \mathrm{~Hz}$ probe tone.

\section{ESRT measurements}

Step 1: The recipient's processor was connected to the programming Pod.

Step 2: The Immittance probe $(226 \mathrm{~Hz})$ of the electro-acoustic impedance equipment was placed in the non- implanted ear (contralateral earphone or probe is not required).

Step 3: The Immittance meter was set to reflex decay mode and a 10$15 \mathrm{msec}$ time window was set.

Step 4: Electrode number 1 was selected and stimulation was begun 10 CLs below the obtained behavioural C-level.

Step 5: Through the reflex decay mode on the immittance equipment, 2-3 stimuli were presented while observing for negative deflection on the immittance equipment.

Step 6: Current level was increased in steps of 5CLs until a response was observed, or until the recipient indicated that the sound is too loud. While stimulating above obtained C-levels, special care was taken for younger children like continuous monitoring of their facial expression to observe any signs of discomfort.

Step 7: Once a response was observed, current Level was decreased in 2CL steps until the response disappeared. The minimum level at which response was observed was taken as the ESR threshold and recorded in the data collection sheet.

Consecutively electrode number 11 and electrode number 22 were selected and ESRT was obtained in similar manner as with electrode number 1 .

\section{Statistical analysis}

For the statistical analysis SPSS version 20.0 was used. The data was subjected to statistical analysis to find out if there was a correlation between two variables across the selected electrodes and significant difference between two groups.

a) A normality check was done using Kolmogorov- Smirnov and Shapiro- Wilk tests.

b) Data was also subjected for Q-Q plots and Bar graph for visualization of distribution.

c) Linearity check was done through Scatter plots.

d) Pearson Product Moment Correlation was applied to obtain the correlation between two variables i.e. C-level \& ESRT for each electrode for both the groups. 
e) Fisher's z test was applied to compare the correlations obtained for the two age groups. The level of significance was set at 0.05 .

\section{Results}

\section{Group A:Younger Children}

Descriptive analysis findings for Group A are shown in Table 2. Tests of Normality i.e., Kolmogorov-Smirnov and Shapiro-Wilk were applied to check the type of distribution. Results of both the normality tests revealed that the data for C-level as well as ESRT followed a normal distribution $(\mathrm{p} \geq 0.05)$ for all electrodes. The Pearson Product Moment Correlation coefficient ( $\mathrm{r}$ ) findings showed that the correlation between $\mathrm{C}$-level and ESRT was significant $(\mathrm{p}<0.05)$ for all electrodes. However, the magnitude of the relationship was not the same. It was strong $(\mathrm{r}=0.852)$, moderate $(\mathrm{r}=0.618)$ and very strong $(\mathrm{r}=0.929)$ for electrode number $1,11, \& 22$ respectively. It is thus seen that higher C-level was associated with higher ESRT. Conversely, lower C-level was associated with lower ESRT.

\section{Group B: older children}

Descriptive analysis findings for Group B are given in Table 3.

Here again, the tests of Normality i.e., Kolmogorov- Smirnov and Shapiro-Wilk revealed that the distributions of C-level as well as ESRT were normal for older children ( $\mathrm{p} \geq 0.05$ ) for all electrodes, except for the C-level of electrode 11 as both test results were significant $(\mathrm{p}<.05)$. This indicates serious deviation of the distribution from normality. So, the distribution was further inspected in detail to identify the cases causing non-normality. The coefficient of skewness and kurtosis of the distributions were calculated. The calculated coefficient of skewness is -2.195 and indicates serious negative skewness in the distribution. However, it is not below ' -2.5 '. The box-plot also did not reveal any outlier. In view of these observations and the smaller sample size, it was decided to ignore the skewness in the data. The coefficient of kurtosis is well within the limit of \pm 2.5 , hence, is not serious to influence normality and mean. Therefore, the distribution of C-level for electrode 11 could be treated normal for Group B.

Table 2 Descriptive statistics for Comfort Levels and ESRT for Group A

\begin{tabular}{lllllll}
\hline & Electrode I & \multicolumn{3}{c}{ Electrode II } & Electrode 22 \\
\hline C levels & ESRT & C levels & ESRT & C levels & ESRT \\
\hline Mean (CL) & 196.6 & 212.7 & 191.7 & 208.15 & 175.55 & 190.55 \\
Variance & 199 & 214 & 193 & 209 & 176 & 188 \\
SD & 184.57 & 142.64 & 156.75 & 185.61 & 173.63 & 261.52 \\
Std. Error & 13.59 & 11.94 & 12.52 & 13.62 & 13.18 & 16.17 \\
Minimum & 3.04 & 2.68 & 2.8 & 3.05 & 2.95 & 3.62 \\
Maximum & 165 & 188 & 168 & 183 & 157 & 168 \\
Range & 219 & 234 & 216 & 229 & 206 & 226 \\
Interquartile range & 15 & 46 & 48 & 46 & 49 & 58 \\
Skewness & -0.594 & -0.198 & 0.018 & -0.219 & 0.717 & 0.607 \\
Kurtosis & 0.313 & -0.148 & -0.626 & -1.098 & 0.164 & -0.136 \\
\hline
\end{tabular}

Table 3 Descriptive statistics for Comfort Levels and ESRT for Group B

\begin{tabular}{lllllll}
\hline & Electrode I & \multicolumn{3}{c}{ Electrode II } & \multicolumn{3}{c}{ Electrode 22 } \\
\hline Mean (CL) & C levels & ESRT & C levels & ESRT & C levels & ESRT \\
Median & 198.3 & 209.05 & 197.2 & 206.35 & 182.15 & 192.35 \\
Variance & 201 & 211.5 & 201.5 & 208.5 & 184 & 190.5 \\
SD & 174.96 & 102.89 & 122.06 & 61.86 & 137.08 & 146.35 \\
Std. Error & 13.23 & 10.14 & 11.05 & 7.86 & 11.71 & 12.09 \\
Minimum & 2.96 & 2.27 & 2.47 & 1.76 & 2.62 & 2.71 \\
Maximum & 165 & 180 & 171 & 187 & 165 & 175 \\
Range & 218 & 228 & 211 & 221 & 213 & 220 \\
Interquartile range & 18 & 48 & 40 & 34 & 48 & 45 \\
Skewness & 53 & 11 & 14 & 11 & 16 & 17 \\
Kurtosis & -0.802 & -0.886 & -1.112 & -0.517 & 0.658 & 0.691 \\
\hline
\end{tabular}


The Pearson Product Moment Correlation coefficient (r) result showed that the correlation between C-level and ESRT was significant for all the electrodes of older children $(p<0.05)$. However, the magnitude was very strong $(r=0.918)$, strong $(\mathrm{r}=0.827)$, and strong $(\mathrm{r}=0.804)$ for electrode 1,11 and 22 respectively. It is thus seen that higher C-level was associated with higher ESRT. Conversely, lower C-level was associated with lower ESRT.

\section{Comparison between the groups: Group A Vs. Group \\ B}

In order to find out whether the correlation is significantly different for the younger and older age groups for the three electrodes Fisher's $\mathrm{z}$ test (1925) was applied. Test findings showed that:

a) The difference in the magnitude of the two correlation coefficients $(-0.066)$ for electrode number 1 was not significant (Fisher's $\mathrm{z}=-0.9118$, $\mathrm{p}$-value $=0.3619$ ). In general, the magnitude of the relationship observed between C-level and ESRT was similar for electrode 1 across two age groups.

b) The difference in the magnitude of the two correlation coefficients $(-0.149)$ for electrode number 11 was not significant (Fisher's $\mathrm{z}=-1.030$, $\mathrm{p}$-value $=0.303$ ). In general, the magnitude of the relationship observed between behaviorally obtained C-levels and ESRT for electrode 11 was similar across two age groups.

c) The difference in the magnitude of the two correlation coefficients $(0.125)$ for electrode 22 was not significant (Fisher's $z=1.5779$, $\mathrm{p}$-value $=0.1146$ ). In general, there is no statistically significant difference in the correlation coefficients of C-level and ESRT for electrode 22 between younger and older children.

\section{Discussion}

Profiling of behavioural C-level and ESRT findings showed that ESRT findings were consistently higher for all the electrodes than behaviorally obtained C-levels. This finding is in agreement with results obtained by de Andrade et al., ${ }^{9}$ who conducted cross-sectional analytical study on $24 \mathrm{CI}$ volunteers and demonstrated that all the selected electrodes $(1,6,11 \& 16)$ for the study showed higher mean ESRT value than their mean C-level counterparts. A similar result was seen in a study done by Hodges et al. ${ }^{10}$ in which they studied electrically elicited middle ear muscles reflexes (eMEMRs) in 25 post linguistically deafened adult CI users with the purpose of examining the clinical reliability of eMEMRs and to determine the various levels of perception. They obtained a correlation coefficient value of $\mathrm{r}=0.91$ (0.0001) which suggested that eMEMR threshold has great predictive value for proving the comfort level. They found that the reflex tended to occur slightly above the voluntary obtained C-level, but reflex threshold never exceeded the level of discomfort. Maps made with C-levels obtained by eMEMR thresholds resulted in speech perception performance that also matched behaviorally derived maps in the adults tested.

In the present study, results of correlation findings revealed that correlation between C-level and ESRT varies from moderate to very strong i.e. 0.68 to 0.93 in younger children whereas for older children it varies in the range of strong to very strong i.e. 0.80 to 0.92 . These results are supported by Stephan et al., ${ }^{11}$ who studied 6 experienced COMBI 40 implant system users. They studied electrically elicited stapedius reflex as well as loudness scaling procedure separately. They found that the overall correlation between ESRT and MCL was high i.e. $r=0.92$ and concluded that the ESRT findings can be used successfully for the programming of speech processor. Their loudness scaling findings also revealed that despite using higher level of current for ESRT, overstimulation was avoided effectively.

Results of the present study also revealed that there was no statistically significant difference between the correlations for the 2 groups for the tested electrodes i.e. electrode 1 (high frequency), electrode 11 (mid-frequency) and electrode 22 (low frequency). A similar research was conducted by Spivak et al., ${ }^{12}$ in 1994. They included a total of 35 patients i.e. 16 children and 19 adults implanted with Nucleus 22 channel cochlear implant. Children ranged in age from 5-12 years whereas adults ranged in age from 21 to 70 years. They tested all the electrodes for behavioural comfort level and ESRT and found that mean difference scores were 19.4 units $(\mathrm{SD}=15.5)$ for adults and 9.6 units $(\mathrm{SD}=10.8)$ for children. The mean scores and standard deviations were calculated without regard to positive or negative sign as in many instances the obtained value of behaviorally measured loudness levels exceeded the obtained ESRT value. The mean values in their study suggested a good agreement between ESRT and C-levels for both groups. On the inspection of individual data, they found that it varied considerably among subjects, with close agreement between C-level and ESRT for four adults and eight children. Remaining 12 subjects' ESRT had significantly over stimulated or under stimulated scores of C- levels but did not exceed the UCL.

Another study was conducted by Greisiger et al., ${ }^{13}$ for the analysis of behavioural and objective measures on a clinical population of various age groups i.e. 0-2, 2-5, 5-10, 10-20 and above 20 years. Intra-operative as well as post-operative measures were utilized for all the age groups. These measures were behavioural $\mathrm{T}$ and $\mathrm{C}$-levels, impedance, ECAP and ESRT. They found that there was weak correlation between visually observed ESRT during intra-operative and T\&C-levels for initial programming levels. Most significant $\mathrm{R}^{2}$ value (0.17) was seen for electrode number 6; age group 5-10 years, after at least one year of CI usage. This weak correlation between intraoperative ESRT and post-operative C-level is supported by Baysal et al., ${ }^{14}$ who studied correlation between Intra and postoperative ESRT in 65 paediatric cochlear implant users who were congenitally deaf and found that intra operative ESRTs were not able to predict the findings of early post-operative ESRTs.

In the present study gross visual inspection of data of individual group reveals that the there is a trend in the current levels required to obtain the C-level as well to elicit the ESRT. Group A inspection reveals that required mean current level decreases from apically placed electrode 22 to basal electrode 1 i.e. 212.7 to $190.6 \mathrm{CLs}$ for ESRT and 196.6 to 175.6 CLs for behavioural C-levels. Similar findings were observed for Group B. These findings are similar to reports from Battmer et al., ${ }^{15}$ who examined 25 adults implanted with Clark/Nucleus device for at least 3 months. They found that stapedial reflexes can be elicited electrically more reliably and require lower intensities from more apical region of cochlea than from the basal part of the cochlea.

In the present study $226 \mathrm{~Hz}$ probe tone was selected for the measurement of ESRT as this probe tone frequency is easily available in all audiology clinics. There are different studies in literature stating that the use of standard $226 \mathrm{~Hz}$ probe tone is not ideal for the measurement of ESRT. According to Wolfe et al., ${ }^{16}$ the use of higher 
probe tone frequencies i.e. 678 or $100 \mathrm{~Hz}$, elicits ESRT at lower current levels than standard $226 \mathrm{~Hz}$. It could be due to this reason that for all the studied electrodes current levels required to elicit ESRT were consistently higher than C-levels. The inclusion criteria for the selection of all the candidates were strictly set at post one year of switch on in the present study. This strict criterion was due to the unstable nature of programming levels i.e. MAP during initial period which gets stabilized after one year and usually needs only minor modifications, ${ }^{13,17}$ and electrode impedances are also stabilized during this period. ${ }^{18,19}$

\section{Conclusions}

Based on the results, the following conclusions can be drawn from study:

i. Higher level of current was required for the elicitation of ESRT than C- levels.

ii. For younger children, the correlations for different electrodes vary in the range of moderate $(0.68)$ to very strong $(0.93)$.

iii. For older children, the correlations for different electrodes vary in the range of strong $(0.83)$ to very strong $(0.92)$.

iv. There was no statistically significant difference seen between the correlation coefficients of both groups.

v. On inspection of individual data, we found that difference in current levels between ESRT and C-level varies from minimum 3 $\mathrm{CL}$ to $41 \mathrm{CLs}$ for younger group whereas it ranged from $2 \mathrm{CL}$ to 30 CLs for older children.

\section{Implication of the study}

a) ESRT could be utilized for setting the C-level during programming of cochlear implantation in cases who are unable to provide reliable behavioural responses, as it lies between most comfortable level and UCL.

b) Correction factors can be utilized to calculate the C-level from ESRT for uncooperative children.

\section{Acknowledgments}

We would like to acknowledgent the participants, their parents.

\section{Conflict of interest}

Authors declare that there is no conflict of interest.

\section{Funding}

None.

\section{References}

1. The Cochlear Implant: An Overview. Indian Speech and Hearing Association, ISHA monograph. 2013;13(2):57.
2. Zwolan TA. Cochlear Implants. In: Katz J, editor, Handbook of Clinical Audiology. 5th edn, USA: Lippincott Williams \& Wilkins; 2002; p. $741-42$.

3. Mertes J, Chinnici J. Cochlear implants- consideration in programming for the pediatric population. Audiology Online. 2006.

4. Brown CJ, Unghes ML, Luk B, et al. The relationship between EAP and EABR thresholds and levels used to program the Nucleus 24 speech processor: Data from adult. Ear Hear. 2000;21(2):151-163.

5. Gross A. Fitting techniques for the pediatric cochlear implant patient. Audiology Online. 2003.

6. Stach BA. Clinical Audiology: An introduction. London: Singular Publishing Group; 1998; p.270.

7. GSI Tympstar ProTM-clinical middle ear analyzer. Grason-Stadler, 10395 West 70th Street, Eden Prairie, Minnesota. Custom Sound 5.1, Cochlear Pvt Ltd, Australia: Cochlear; 2018.

8. de Andrade KCL, Muniz LF, Menezes PL, et al. The Values of Electrically Evoked Stapedius Reflex in Determining the Maximum Comfort Level of a Cochlear Implant. J Am Acad Audiol. 2018;29(4):292-99.

9. Hodges VA. Electrical middle ear muscle reflex: Use in cochlear implant programming. Otolaryngol Head Neck Surg. 1997;117(3):255-261.

10. Stephan K, Muller WK. Post-operative stapedius reflex tests with simultaneous loudness scaling in patients supplied with cochlear implants. Audiology. 2000;39(1):13.

11. Spivak LG, Chute PM. The relationship between electrical acoustic reflex thresholds and behavioural comfortable levels in children and adult cochlear implant patients. Ear Hear. 1994;15(2):184-192.

12. Greisiger R, Shallop JK, Hol PK, et al. Cochlear implantees: Analysis and behavioural measures for a clinical population of various age groups. Cochlear Implants Int. 2015;16(S4):1-19.

13. Baysal E, Karatas E, Deniz M, et al. Inta-and postoperative electrically evoked stapedius reflex thresholds in children with cochlear implants. Int journal of Pediatric otorhinolaryngoloy. 2012;76: 649-652.

14. Battmer RD, Laszig R, Lehnhardt E. Electrically elicited stapedius reflex in cochlear implants patients. Ear Hear. 990;11(5):370-374.

15. Wolfe J, Gilbert M, Schafer E. et al. Optimizations for the electricallyevoked stapedial reflex threshold measurement in cochlear implant recipients. Ear Hear. 2017;38(2):255-261.

16. Vargas JL, Sainz M, Roldan C, et al. Long term evolution of the electrical stimulation levels for cochlear implant patients. Clin Exp Otorhinolaryngol. 2012;5(4):194-200.

17. Henkin Y, Neeman KR, Muchnik C, et al. Changes over time in electrical stimulation levels and electrical impedance values in children using the Nucleus $24 \mathrm{M}$ cochlear implant. Int otorhinolaryngol. 2003;67(8):873-880.

18. Zadrozniak M, Szymanski M, Siwiec H, et al. Impedance changes in cochlear implant users. Otolaryngol Pol. 2011;65(3):214-217. 